\title{
Efficient Method for Predicting Crystal Structures at Finite Temperature: Variable Box Shape Simulations
}

\author{
Laura Filion, Matthieu Marechal, Bas van Oorschot, Daniël Pelt, Frank Smallenburg, and Marjolein Dijkstra \\ Soft Condensed Matter, Debye Institute for NanoMaterials Science, Utrecht University, Princetonplein 5, The Netherlands \\ (Received 27 July 2009; published 29 October 2009; publisher error corrected 30 October 2009)
}

\begin{abstract}
We present an efficient and robust method based on Monte Carlo simulations for predicting crystal structures at finite temperature. We apply this method, which is surprisingly easy to implement, to a variety of systems, demonstrating its effectiveness for hard, attractive, and anisotropic interactions, binary mixtures, semi-long-range soft interactions, and truly long-range interactions where the truly long-range interactions are treated using Ewald sums. In the case of binary hard-sphere mixtures, star polymers, and binary Lennard-Jones mixtures, the crystal structures predicted by this algorithm are consistent with literature, providing confidence in the method. Finally, we predict new crystal structures for hard asymmetric dumbbell particles, bowl-like particles and hard oblate cylinders and present the phase diagram for the oblate cylinders based on full free energy calculations.
\end{abstract}

The ability of atomic, colloidal, and nanoparticle systems to self-organize into crystal structures makes the prediction of stable crystal structures in these systems an important challenge for science. In a recent Review article, Woodley and Catlow claim "The prediction of structure at the atomic level is one of the most fundamental challenges in condensed matter science" [1]. It is therefore not surprising that the subject has received much attention from the scientific community over the last several decades.

The question itself is deceivingly simple: assuming that the underlying interactions between constituent particles are known, which crystal structures are stable? Before the 1990s most of our understanding of atomic crystal structures came from simple empirical rules, now well documented in solid state text books. In 1990, Pannetier et. al. proposed a method based on simulated annealing techniques [2]. In their method a general crystal structure was described in terms of lattice and basis vectors, and the "cost" function for the system was minimized using simulated annealing. The method can easily be extended to any atomic system for which a suitable cost function, e.g., the potential energy, can be constructed [3]. Recently, more advanced minimization techniques such as genetic algorithms (GAs) (e.g., [4,5]) and Monte Carlo (MC) basin hopping algorithms [6] have been applied. Typically these techniques are used to locate the minimum potential energy of the system, and as such, probe the zero-temperature phase behavior. However, for systems where the entropy plays a significant role, these techniques break down. For instance, new crystal structures can appear in the phase diagram at finite temperature, which are different from the zero-temperature crystal structures, and hence predicting the zero-temperature structures will not be sufficient for making predictions at finite temperature. Additionally, for hard systems the potential energy is always zero as only nonoverlapping configurations contribute to the partition function, and hence, crystal structures are stabilized by entropy alone. For such systems it is difficult to construct an appropriate cost function, and therefore the MC basin hopping algorithm and GAs cannot be applied to hard-core systems. With the recent progress in the chemical synthesis and fabrication of colloidal particles and nanoparticles, there is unprecedented ability to control the interparticle interactions in such systems, through particle synthesis and chemistry [7], through the solvent in which they are dispersed [8], and through the application of external fields [9]. The resulting interactions between the colloidal particles can be made sufficiently weak that emerging crystal structures are stabilized predominantly by entropic contributions to the free energy. For instance, experimental realizations of binary hard-sphere mixtures and hard bowl-like particles as described in this Letter, are stabilized purely by entropy. Additionally, crystal structures found in binary mixtures of nanoparticles (e.g., $\mathrm{NaCl}, \mathrm{AlB}_{2}$, $\mathrm{NaZn}_{13}$ ) are consistent with hard-sphere interactions, indicating a significant entropic contribution to the free energy of these systems [10]. Hence, locating stable crystal structures for soft interactions at finite temperature and for hard-core interactions presents an important and exciting challenge and plays a vital role in the rational design of advanced materials.

To the best of our knowledge, there have been only two attempts to predict crystal structures for such systems, an ergodicity search algorithm [11] and the ParrinelloRahman metadynamics method [12]. In the ergodicity algorithm, the entropic contribution to the free energy is approximated by the harmonic phonon contribution. However, even such an approximation is difficult and computationally expensive, and not straightforward to apply to hard-core interactions. In contrast, metadynamics 
uses a constant pressure molecular dynamics (MD) simulation with variable box shape, to study phase transitions between various crystal structures. In this case, the free energy is intrinsically included in the model. We are, however, unaware of any attempts to apply metadynamics to predicting crystal structures out of the fluid phase.

In this Letter we present a novel method to predict crystal structures at finite temperature for a wide variety of systems, including hard-core systems whose phase behavior is purely entropy-driven such as binary sphere mixtures, asymmetric dumbbells, bowls, and platelets.

The general algorithm consists of MC simulations in an isotension-isothermal ensemble and is similar in spirit to the Parrinello-Rahman metadynamics method [12]. However, there are a few important differences, described in more detail below. First, we extend the ParrinelloRahman MD method which is based on the isotension ensemble to MC simulations, for which the constant stress condition is much easier to implement. Second, we apply our method to predict crystal structures out of the fluid phase. To this end, the initial pressure is chosen to be below the fluid to solid transition and the pressure is increased incrementally until the system solidifies. We note, however, that we can also use our method to study crystal transformations similar to the metadynamics method [12], which can serve as a stability check of a crystal structure; e.g, the cuboctahedral $A B_{13}$ structure melts into the isostructural $A B_{13}$ structure, and the $\mathrm{Ag}_{2} \mathrm{Se}$ structure, predicted in a GA, melts into the stable $\mathrm{MgZn}_{2}$. In our MC simulations, lattice vectors $\vec{L}$ comprise the simulation box, and the positions of the particles in the crystal; i.e., the basis vectors $\vec{R}$, are expressed in terms of these lattice vectors. As is typical for a MC simulation in the isothermal-isobaric (NPT) ensemble, each MC step consists of either a trial particle displacement or a trial volume change where the acceptance rules of the particle and volume moves are given by the Metropolis algorithm [13]. In order to allow for box shape fluctuations, a trial volume move involves an attempt to change the orientation and the length of a random lattice vector. Third, an important novel part of our algorithm is to treat the simulation box as a unit cell, and as such work with extremely small particle numbers. In this Letter, the number of particles in the simulation box ranges from 1-12. Working with small simulation boxes, and allowing the shape of the simulation box to fluctuate introduces new problems. The main problem is that while the system is in the liquid phase, the shape of the box fluctuates significantly. Thus, the box can become extremely distorted, which makes the potential energy summation time consuming. To avoid this problem, we use the lattice reduction technique described in Ref. [5] to redraw the unit cell when it becomes too distorted. Additionally, we impose a restriction on all the angles and the lengths of the lattice vectors to avoid trivial unphysical crystal structures. Without these restrictions the particles tend to line up in columns, such that the particles only interact with their own periodic images in one of the lattice directions resulting in unphysical contributions to the entropy. Angles less than $30^{\circ}$ and greater than $150^{\circ}$ are not accepted. Such a condition prevents the box (particularly while in the fluid phase) from an extreme distortion, while allowing for all possible crystal phases to emerge in our simulations. Additionally, for each configuration of interest, we run several parallel MC runs, with various starting configurations and pressures.

We applied this method to several representative systems: binary hard-sphere mixtures, binary Lennard-Jones mixtures, star polymers, dipolar hard spheres in an electric field, asymmetric hard dumbbells, hard bowl-like particles, and hard platelets.

In the case of binary hard-sphere mixtures, the initial pressure was chosen such that the simulation started in the fluid phase. The pressure was increased slowly until the particles crystallized, and the crystal structure remained constant. In order to facilitate the identification of the crystal structure, the pressure was then increased significantly. The results for $A B$ and $A B_{2}$ crystal structures are shown in Table I for varying size ratios $\alpha=\sigma_{S} / \sigma_{L}$ with $\sigma_{L, S}$ the diameter of the large and small hard spheres, respectively. The close packed structures for $A B[14,15]$ and $A B_{2}$ [14] stoichiometry have been predicted previously, using a GA [14] and a simulated annealing technique using structures from a crystallographic database [15]. We note that all the structures predicted in Refs. $[14,15]$ are also predicted by our algorithm. Additionally, the stability of the $A B$ and $A B_{2}$ structures has been determined in computer simulations using free energy calculations.

TABLE I. $A B$ and $A B_{2}$ structures predicted for binary hardsphere mixtures for various size ratio $\alpha=\sigma_{S} / \sigma_{L}$. Each simulation was repeated between 10-20 times with different initial configurations, and the frequency (\%) of the resulting structures is listed. * has been used to denote structures which are slightly distorted from their symmetric structure. Structures which have been previously predicted to be stable [16] are indicated in bold. For $\alpha=0.4$ and 0.7 , our method was unable to predict ordered $A B_{2}$ crystal structures, which is consistent with the fact that no $A B_{2}$ structures are stable for these size ratios.

\begin{tabular}{ccccc}
\hline \hline$\alpha$ & Structure $A B(2: 2)$ & $\%$ & Structure $A B_{2}(4: 8)$ & $\%$ \\
\hline 0.4 & NaCl & $40 \%$ & & \\
& NiAs & $20 \%$ & & \\
& SG 166* & $30 \%$ & & $55 \%$ \\
0.5 & SG 166 & $60 \%$ & AlB $_{2}$ & \\
& CrB & $30 \%$ & & $40 \%$ \\
0.6 & CrB & $70 \%$ & AlB $_{2}$ & \\
& SG 166 & $10 \%$ & & \\
& SG 12 & $10 \%$ & & \\
0.7 & CsCl & $40 \%$ & & \\
& $\mathrm{CrB}$ & $20 \%$ & & \\
0.8 & $\gamma \mathrm{CuTi}$ & $30 \%$ & Laves & \\
& $\alpha \mathrm{IrV}$ & $10 \%$ & SG139 & \\
& $\mathrm{CsCl}$ & $10 \%$ & & \\
\hline \hline
\end{tabular}


These studies show that $\mathrm{NaCl}$ is stable for $\alpha \in$ [0.2-0.42], $\mathrm{AlB}_{2}$ for $\alpha \in[0.42-0.59]$, and the Laves phases are stable for $\alpha \in[0.74-0.84]$ [16]. We indeed observe from Table I that the stable crystal structures, which are denoted in bold, are indeed predicted by our new method.

For $\alpha=0.5$ we see that the most frequently occurring $A B$ crystal structure is not the best packed structure $\mathrm{CrB}$, but rather a crystal structure within the symmetry group 166 (denoted SG 166). A cartoon of this structure along with $\mathrm{AlB}_{2}$ is shown in Fig. 1 . While $\mathrm{AlB}_{2}$ is stable for $\alpha=$ 0.5 , no $A B$ stoichiometry crystal structure is found to be stable. An examination of SG 166 shows that while it has a different stoichiometry than $\mathrm{AlB}_{2}$, it has similar planes, i.e., the small colloids sit on a 2D honeycomb lattice, while the larger colloids are in hexagonal planes. Thus it appears that while the system was unable to find the structure with the lowest free energy since the stoichiometry was incorrect, it located a compromise.

The crystal structures appearing at $\alpha=0.8$ are also of significant interest. At this size ratio the Laves phases are expected to be stable. Our results show that a significant majority of our simulations have resulted in Laves phases $(75 \%)$. It is interesting to note that we have been able to improve the results for this size ratio dramatically by restricting the length of the lattice vectors. When this restriction is removed, we only find 30\% resulting in the Laves phases. Additionally, for all $\alpha$, the runs with 12 particles per unit cell do not produce crystal structures when this restriction is removed. Summarizing, we find that our method predicts the stable crystal structures for binary hard-sphere mixtures, including a few additional structures which are the best packed crystal structures for the given stoichiometry, and structures whose planes were related to stable crystal structures.

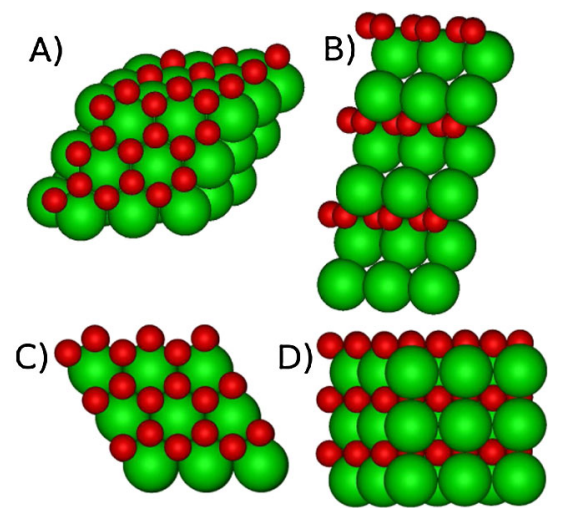

FIG. 1 (color online). (a) and (b) correspond, respectively, to the $x y$ and $x z$ faces of the $A B$ crystal structure from Space Group 166 found in the set of predicted phases for size ratios 0.4-0.6. (c) and (d) correspond to the $x y$ and $x z$ phases of the $\mathrm{AlB}_{2}$ crystal structure. Note that the planes made by the small particles in both structures correspond to $2 \mathrm{D}$ honeycomb planes, while the large colloids sit on a hexagonal lattice.
A recent MD simulation demonstrated that the Wahnström binary Lennard-Jones mixture, which has often been used in simulations to study glassy behavior, crystallizes spontaneously into the $\mathrm{MgZn}_{2}$ Laves phase [17]. The Lennard-Jones interaction is given by $u\left(r_{i j}\right)=$ $4 \epsilon_{i j}\left[\left(\sigma_{i j} / r_{i j}\right)^{12}-\left(\sigma_{i j} / r_{i j}\right)^{6}\right]$, where the Lorentz-Berthelot mixing rule $\sigma_{A B}=\left(\sigma_{A A}+\sigma_{B B}\right) / 2$ was used. Choosing the parameter $\epsilon_{A A}=\epsilon_{B B}=\epsilon_{A B}=1$, and $\sigma_{B B} / \sigma_{A A}=$ $0.8-0.84$, we have also located predominantly the $\mathrm{MgCu}_{2}$ and $\mathrm{MgZn}_{2}$ Laves phases, which is consistent with Ref. [17]. We also applied the method to study star polymers [18], which interact with an extremely soft interaction without a hard core. The interaction between star polymers is characterized by the arm number $f$ and the corona diameter $\sigma$. For this system, we only allowed the shape of the box to fluctuate and studied the system at fixed density. We predicted the crystal structures appearing for $f=64$ and $\sigma=1$ as a function of the density. We observed that a single MC simulation at constant density visited a large range of (meta)stable crystal structures. In this case, for each run we saved the crystal structures with the lowest potential energy, i.e., face-centered cubic (fcc), trigonal, diamond, hexagonal, and hexagonal-close-packed (hcp) crystal structures upon increasing density which all agree with Ref. [5]. We also implement box shape moves in a system with long-range interactions treated using Ewald sums, i.e., a system of dipolar hard spheres in an electric field. The interaction potential is given by $\beta u(\mathbf{r})=$ $(\gamma / 2)(\sigma / r)^{3}[1-3 \cos (\theta)]$ with $\gamma=15$. The phase behavior of this system has been studied previously [19], and the three known stable crystal structures are the bodycentered-tetragonal (bct), fcc, and hcp phase. The crystal phases predicted by our algorithm included bct, fcc, and hcp phases and are consistent with previous phase diagram calculations [19], thereby demonstrating that our method can be applied to systems with long-range interactions.

Having established the reliability of our method as all the predicted structures are consistent with the literature, we now apply the algorithm to previously unexplored systems such as hard asymmetric dumbbells, hard bowllike particles and hard platelets. In the case of asymmetric dumbbell particles consisting of a tangent large and small hard sphere, our method predicts crystal structures that are atomic analogs of $\mathrm{NaCl}, \mathrm{CsCl}, \gamma \mathrm{CuTi}, \mathrm{CrB}$, and $\alpha \mathrm{IrV}$ when we regard the two individual spheres of each dumbbell independently. The bonds that connect the dumbbells appear to connect random small-large pairs, and hence the crystal structures are in fact aperiodic in positional and orientational order. These results are consistent with the structures found for the $A B$ structures of the binary hardsphere mixtures. The bowl-like particles are modeled by the solid of rotation of a crescent, where the thickness of the crescent is denoted by $D$, the diameter by $\sigma$, and the axis of rotation is defined as $\mathbf{u}$. To predict the crystal structures, unit cells of 2 to 6 particles were examined. The system was initialized at a pressure of $\beta p \sigma^{3}=10$ and 


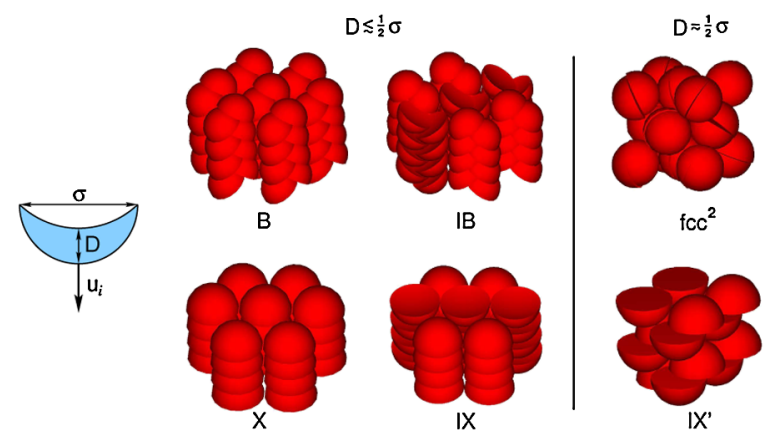

FIG. 2 (color online). Cartoon and predicted crystal structures for hard bowls.

was increased by a factor of 10 each step until a pressure of $\beta p \sigma^{3}=10^{6}$ was reached. The resulting structures are shown in Fig. 2. Subsequent free energy calculations showed that four of the six predicted crystal structures are indeed stable: IB, IX', IX and $\mathrm{fcc}^{2}$ [20]. Finally, we apply this method to hard cusp-free platelets [21], which we model by oblate spherocylinders [see Fig. 3(a)]. Our method finds the two crystal phases, $X_{\text {tilted }}$ and $X_{\text {aligned }}$, depicted in Fig. 3. The particles are tilted in the $X_{\text {tilted }}$ phase with respect to each other. Surprisingly, no crystal analog exists for cut spheres [22], which is the more conventional model for platelets. $X_{\text {aligned }}$ is the crystal phase also found for cut spheres, where all particles point in the same direction. The phase diagram for this system is presented in Fig. 3. Interestingly, both predicted crystal structures are stable in some region of the phase diagram, specifically, $X_{\text {titled }}$ for $L / D \lesssim 0.46$ and $X_{\text {aligned }}$ for larger $L / D$. We note that this phase diagram is based on
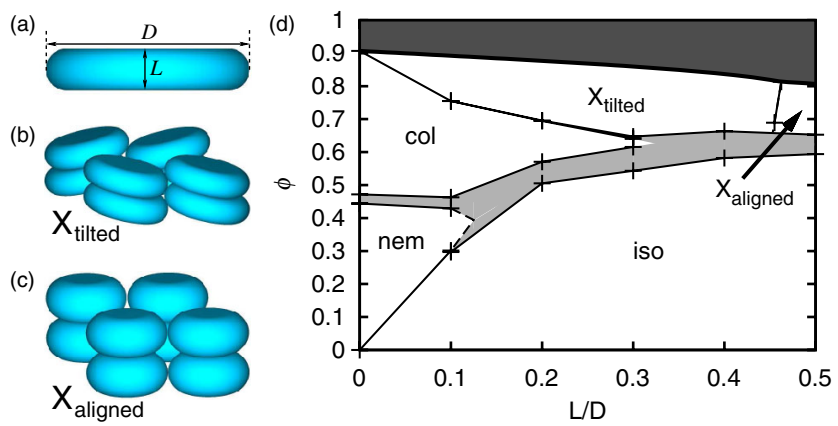

FIG. 3 (color online). Phase diagram of hard oblate spherocylinders in the packing fraction $(\phi)$ versus dimensionless thickness $(L / D)$ representation (d), where the diameter $D$ and thickness $L$ are depicted in (a). The state points in the dark gray area are inaccessible since they lie above the maximum close packing line. $X_{\text {aligned }}$ and $X_{\text {tilted }}$, denote the aligned and tilted crystal structures as shown in (b) and (c), "iso," "nem," and "col" are the isotropic, nematic, and the columnar phase, respectively. The lines are a guide to the eye. The coexistence packing fractions of the nematic and the columnar phase for $L / D=0$ are from Ref. [24]. full free energy calculations, which will be explained elsewhere [23].

In conclusion, we have presented an algorithm to predict the crystal structures at finite temperature for a wide variety of systems. In all cases our algorithm has predicted the stable crystal structures, along with a small number of additional phases. The algorithm is extremely simple to implement, and yields results consistent with the known phase behavior. We expect this algorithm to be widely applicable in the study of new materials, such as colloidal particles, nanoparticles, and micelles.

We would like to thank A.-P. Hynninen for the original idea behind this method. Financial support from NWO-VICI and High Potential programme (UU) is acknowledged.

[1] S. M. Woodley and R. Catlow, Nature Mater. 7, 937 (2008).

[2] J. Pannetier et al., Nature (London) 346, 343 (1990).

[3] H. R. Karfunkel and R. J. Gdanitz, J. Comput. Chem. 13, 1171 (1992).

[4] A. R. Oganov and C. W. Glass, J. Chem. Phys. 124, 244704 (2006); D. P. Stucke and V.H. Crespi, Nano Lett. 3, 1183 (2003).

[5] D. Gottwald et al., J. Chem. Phys. 122, 204503 (2005).

[6] D. J. Wales and H. A. Scheraga, Science 285, 1368 (1999); D. J. Kraft et al., J. Am. Chem. Soc. 131, 1182 (2009).

[7] V. N. Manoharan et al., Science 301, 483 (2003).

[8] P. Bartlett and A. I. Campbell, Phys. Rev. Lett. 95, 128302 (2005); M. E. Leunissen et al., Nature (London) 437, 235 (2005).

[9] A. Yethiraj and A. van Blaaderen, Nature (London) 421, 513 (2003).

[10] E. V. Shevchenko et al., J. Am. Chem. Soc. 128, 3620 (2006).

[11] J. C. Schon et al., J. Chem. Phys. 128, 194712 (2008).

[12] R. Martoňák et al., Phys. Rev. Lett. 90, 075503 (2003).

[13] D. Frenkel and B. Smit, Understanding Molecular Simulations: From Algorithms to Applications (Academic Press, California, USA, 2002).

[14] L. Filion and M. Dijkstra, Phys. Rev. E 79, 046714 (2009).

[15] J. K. Kummerfeld et al., J. Phys. Chem. B 112, 10773 (2008).

[16] E. Trizac et al., Mol. Phys. 90, 675 (1997); A.-P. Hynninen et al., Nature Mater. 6, 202 (2007); M. D. Eldridge et al., Nature (London) 365, 35 (1993).

[17] S. Toxvaerd et al., J. Chem. Phys. 130, 224501 (2009).

[18] M. Watzlawek et al., Phys. Rev. Lett. 82, 5289 (1999).

[19] A.-P. Hynninen and M. Dijkstra, Phys. Rev. Lett. 94, 138303 (2005).

[20] M. Marechal et al., (to be published).

[21] M. Wojcik and K. Gubbins, Mol. Phys. 53, 397 (1984); A. Cuetos and B. Martinez-Haya, J. Chem. Phys. 129, 214706 (2008).

[22] J. A. C. Veerman and D. Frenkel, Phys. Rev. A 45, 5632 (1992).

[23] M. Marechal et al. (to be published).

[24] M. Bates and D. Frenkel, Phys. Rev. E 57, 4824 (1998). 